2-1-2017

\title{
Management of Forehead Scars
}

\author{
Ryan N. Heffelfinger \\ Thomas Jefferson University \\ Akshay Sanan \\ Thomas Jefferson University \\ Lucas M. Bryant \\ Thomas Jefferson University
}

Follow this and additional works at: https://jdc.jefferson.edu/otofp

Part of the Otolaryngology Commons

Let us know how access to this document benefits you

\section{Recommended Citation}

Heffelfinger, Ryan N.; Sanan, Akshay; and Bryant, Lucas M., "Management of Forehead Scars" (2017). Department of Otolaryngology - Head and Neck Surgery Faculty Papers. Paper 35. https://jdc.jefferson.edu/otofp/35

This Article is brought to you for free and open access by the Jefferson Digital Commons. The Jefferson Digital Commons is a service of Thomas Jefferson University's Center for Teaching and Learning (CTL). The Commons is a showcase for Jefferson books and journals, peer-reviewed scholarly publications, unique historical collections from the University archives, and teaching tools. The Jefferson Digital Commons allows researchers and interested readers anywhere in the world to learn about and keep up to date with Jefferson scholarship. This article has been accepted for inclusion in Department of Otolaryngology - Head and Neck Surgery Faculty Papers by an authorized administrator of the Jefferson Digital Commons. For more information, please contact: JeffersonDigitalCommons@jefferson.edu. 


\title{
Management of Forehead Scars
}

\section{AUTHORS}

Ryan Heffelfinger, MD (corresponding author)

Thomas Jefferson University Hospital

Thomas Jefferson University

Assistant Professor

Department of Otolaryngology - Head \& Neck Surgery, Division of Facial Plastic Surgery

925 Chestnut St

7th Floor

Philadelphia, PA 19107

Ryan.Heffelfinger@jefferson.edu

Lucas M Bryant, MD

Thomas Jefferson University Hospital

Thomas Jefferson University

Resident Physician

Department of Otolaryngology - Head \& Neck Surgery, Division of Facial Plastic Surgery

925 Chestnut St

6th Floor

Philadelphia, PA 19107

Dr.lucasbryant@gmail.com

\author{
Akshay Sanan, MD \\ Thomas Jefferson University Hospital \\ Thomas Jefferson University \\ Resident Physician \\ Department of Otolaryngology - Head \& Neck Surgery, Division of Facial Plastic Surgery \\ 925 Chestnut St \\ 6th Floor \\ Philadelphia, PA 19107 \\ Akshay.Sanan@jefferson.edu
}

\section{DISCLOSURES}

None of the authors listed above have anything to disclose.

\section{ACKNOWLEDGEMENTS}

Ryan Rimmer, MD; Resident Physician; Thomas Jefferson University, Department of Otolaryngology Head \& Neck Surgery. Illustration of double hatchet flap (Figures 2, 3, 4)

SYNOPSIS (1-paragraph, < 100 words for PubMed indexing)

This chapter is an overview of scar management within the forehead region. It addresses the unique challenges specific to the treatment of forehead wounds. A logical, step-wise approach is used. A subsite based treatment algorithm is provided along with a review of current best practices. Pertinent case examples are included for demonstration purposes.

KEY WORDS (5-8)

Wound healing, scars, forehead, facial plastic surgery, laser, filler, reconstruction, eyebrow 


\section{KEY POINTS}

- The homogenous topography of the forehead makes the creation of inconspicuous scars challenging.

- Careful pre-operative planning, open communication and setting realistic expectations are vital to any scar revision case.

- Use natural borders (hairline, brow, temporal line, facial rhytids) etc. to disguise scars whenever possible.

- Protection of function always takes priority over restoration of form.

- Convex, contracted scars may benefit from injection of filler, including fat transfer.

- Hair transplant can help restore appearance when scars involve hair bearing skin. 


\section{INTRODUCTION}

Injuries to the forehead pose several challenges to the facial plastic and reconstructive surgeon. Some of these challenges are held in common with other facial regions, while others are unique. This chapter is designed to take the reader through an organized, step-wise approach to acute and chronic scar management of the forehead region. Forehead wounds may present for a variety of reasons, including facial trauma, defects remaining after excision of malignant or non-malignant lesions, the result of congenital facial lesions, and from iatrogenic causes.

Reconstruction of forehead wounds can be approached using a standardized and complete methodology. Repair may range from local wound care, primary closure, local tissue rearrangement, regional flap reconstruction and complex free tissue transfer, or any combination thereof. In addition to optimizing the patient's aesthetic appearance, goals of repair include protection of vital structures, prevention of infection, and avoidance of chronic wound complications.

Successful reconstruction depends on several synergistic factors. The nature of the wound, careful pre-operative planning, surgical competence, and the global medical status of the patient all play a role. When one of these pillars of wound repair are lacking, the others become even more important. Patients who smoke, have vasculopathy, or are not medically stable to undergo an intensive procedure may have limited options for repair. The importance of this concept cannot be emphasized enough for the neophyte surgeon. Patients may require serial procedures (in the case of tissue expander use for example), and the possibility of this should be discussed up front. Depending on the nature of their injury, patients may remain significantly disfigured, even after the best possible reconstructive effort has been well executed. It is vital the surgeon maintain open and honest communication with the patient throughout the reconstructive journey.

A scar is the final, unavoidable result of any wound; whether it is repaired masterfully, or neglected in its entirety. Facial scarring can be particularly troublesome to patients as it is something the patient and those they encounter will see on a daily basis. Even if vital structures have been protected and function restored, an unappealing facial scar can cause persistent and significant emotional suffering in 
patients (Levine). Surgeons can minimize the impact of facial scarring on a patient through careful planning and the use of optimal reconstructive measures.

Scars are fluid, evolving over time. They change in color when healing and when exposed to various environmental factors such as sunlight or a chemical irritant. They change in shape when exposed to tension or movement during wound healing. Although the strength of a scar builds over time, it will never regain the full resilience of native tissue. Lastly, scars are subject to hereditary forces. Some patient's wound healing may be burdened with the challenges of dyschromias, scar hypertrophy, or keloid reactions over time. The successful facial plastic surgeon uses a broad armamentarium to address the unique needs of each patient and optimize final outcomes.

\section{MANAGEMENT AND TREATMENT}

\section{OVERVIEW}

Scar management begins at the time of cutaneous insult. Wounds should be cleaned, and copious irrigation used. Anesthesia (local, IV, or general) may be needed for adequate debridement and examination. Never allow treatable patient discomfort to limit one's initial examination (Bryant). Depending on the wound class, antibiotics may be indicated. In cases of polytrauma, where the facial wound is not a singular injury, cooperation with several other treatment teams may be necessary. When forehead wounds are the result of a surgical excision, these primary steps may have already occurred.

In comparison to other facial regions, the forehead is relatively devoid of geometric complexity. Any aberration of the symmetric, gentle contour, and homogenous appearance of the forehead readily stands out to the observer. A concave scar within the convex topography of the mid forehead can cast shadows and distort light reflection patterns, making an otherwise innocuous scar readily stand out.

Complete understanding of facial anatomy as well as the biomechanical interactions which occur both acutely and over time within the face is a foundational principle in successful facial reconstruction. One of the first things a surgeon should become facile with facial analysis. Facial analysis helps to define both aesthetic ideals, as well as aberrations. Facial analysis can be performed from many observer views (frontal, oblique, profile), but forehead analysis is often most helpful using the frontal view. One should be 
careful not to perseverate with exacting geometric definitions at the expense of patient preference and the pursuit of global facial harmony.

All forehead interventions must take into consideration the effects it will have on adjacent key anatomic structures. Forehead tissues are generally unforgiving, and distortion of adjacent subsites is a frequent challenge. It is also important to anticipate future changes, such as the effect of balding on hairline shape and location. A carefully placed scar line within a young male's hairline may become painfully obvious several years later.

\section{FACIAL ANALYSIS}

The facial frame can be divided into subunits both vertically and horizontally. Facial height can be divided into thirds, with the forehead comprising the superior third. The superior third (forehead) is defined as running from trichion to glabella. (Figure 1) Although typically described as the superior third, variability in a patient's hairline may result in the forehead subunit comprising significantly more or less than $1 / 3$ of the overall vertical facial dimension.

The face can also be subdivided into into vertical segments using the facial vertical fifths model. This results in three unique vertical subunits: mid-forehead, paramedian, and lateral (temporal). (Figure 1) The lateral forehead subunit is concave in shape. It is bordered by the hairline postero-superiorly and the temporal line and lateral orbital rim anteriorly. The paramedian forehead region is bordered superiorly by the hairline, and inferiorly by the superior orbital rim and overlying brow. The mid-forehead region is bound superiorly by the hairline (including the trichion), and inferiorly by the glabella and nasal root (nasion, sellion \& rhinion).

\section{MID-FOREHEAD}

When using the facial fifths model of facial analysis, the mid-fifth describes the mid-forehead region. It is the only non-paired subunit of the forehead. Its topography is homogenous and it is the most projected subunit of the forehead. The surface anatomy is largely convex in nature. It contains the medial border of both frontalis muscles. The frontalis is the anterior muscle belly of the occipitofrontalis muscle. 
Due to the lack of frontalis muscle in the midline, the mid-forehead region is amenable to either vertical or midline scars. This region contains both horizontal rhytids (frontalis, procerus) and vertical rhytids (corrugator supercilii).

Superior mid-forehead defects may be closed with advancement flaps. Trichophytic incisions, or those placed within the hairline (as would be done for a coronal approach), may be used to gain further tissue laxity. An example of this is an O-T rotational flap to close a superior mid-forehead defect adjacent to the hairline. Midline vertically oriented scars may be less likely to undergo scar widening due to the lack of frontalis muscle activity during healing. Inverted V-Y advancement flaps may help obviate eyebrow medialization with primary closure of glabellar wounds. (Baker)

\section{PARAMEDIAN FOREHEAD}

The paramedian forehead is bordered inferiorly by the superior orbital rim, laterally by the temporal line, and medially by the border of brow/canthus. Notable skeletal landmarks include the prominence of the supraorbital rim. The majority of sensory innervation of the forehead is provided via terminal branches of the ophthalmic division $\left(\mathrm{V}_{1}\right)$ of the trigeminal nerve. The supratrochlear neurovascular bundle lies medial to the supraorbital neurovascular bundle and runs beneath the corrugator supercilii and frontalis muscles. The supraorbital notch, which may be palpated along the superior orbital rim, lies approximately at the junction of the mid and medial thirds of brow. These nerves are paired with the supraorbital and supratrochlear vessels. In most patients, the supraorbital structures exit via a notch. However, in approximately $10 \%$ of the population, the neurovascular structures may exit slightly more superiorly via a true foramen. These patients are at increased risk for injury during dissection. Overlying this is the eyebrow. While eyebrow hairs do not undergo balding patterns intrinsic to scalp hair, the surgeon should consult the patient regarding facial grooming patterns (shaving, plucking, waxing), as this may alter the brow shape and brow line. This area holds several key neurovascular structures, namely the supratrochlear artery and nerve, and the supraorbital artery and nerves. The inferior border of the paramedian region involves periorbital structures including the brow and upper eyelid. 
The degree of bossing of an individual's forehead varies, but the region from the midline to the mid-pupillary line is convex. The relaxed skin tension lines in the forehead are horizontal. When possible, horizontally oriented incisions are preferred to allow maximal scar camouflage. Eyebrow elevation is of particular importance in this forehead region. When large defects $(>3 \mathrm{~cm})$ are closed primarily in a horizontally oriented plane, the result often yields excessive eyebrow elevation. Defects near the hairline in the paramedian forehead that are closed in a horizontal fashion impact the eyebrow much less compared to inferiorly based defects. When defects will effect eyebrow elevation, the surgeon can limit this by suturing the eyebrow dermis and the underlying muscle to the periosteum of the superior orbital rim.

To minimize scar for defects of the paramedian forehead near the hairline, a bipedicle advancement flap is used. Bipedicle flaps are recruited from tissue superior to the defect and the incision is carried full thickness to the galea. The bipedicled flap is well vascularized by the subdermal plexus and provides a hidden scar. One of its drawbacks is lowering of the anterior hairline. Of note, the longer the releasing incision for the bipedicled flap, the greater mobility the flap has. Advancement flaps are used to close skin defects in the paramedian forehead, which are not amenable to primary closure. Incisions are made along the horizontal RSTL's. Flaps are made at a 4:1 ratio of length to width and are dissected in the subcutaneous tissue plane, superficial to the neurovascular bundle. Effective dissection in the correct plane, squaring of the defect borders and removing standing cutaneous deformities is imperative to appropriate scar formation. An effective method to camouflage scar formation is to make the flap slightly wider than necessary in order to hide incisions in nearby skin creases.

Management of scar in the paramedian forehead region can be challenging. Thus, attempts should be made by the surgeon to avoid vertical, curvilinear and oblique incisions because they result in scars that are not in the RSTL's. Majority of defects should be repaired primarily in a horizontal orientation or reconstructed with horizontally oriented unilateral or bilateral advancement flaps. Further, vertically oriented primary repair should be avoided. Rotation and transposition flaps should also be avoided.

In paramedian forehead wounds, which include the brow, additional procedures such as lateral cantholysis may be required to address the integrity of the eyelid. (Mutlu). Other options in the brow region include a pedicled forehead flap (Fricke, Mutlu). If a significant amount of tissue loss occurs in the 
supra-orbital region, reconstruction may result in a similar effect as a direct brow lift. In such cases, it is reasonable to consider addressing the contralateral face to maintain symmetry. Periorbital defects extending below the eyebrow are discussed in a different chapter of this text.

A good method for closure of moderate sized defects within the paramedian and temporal region is the hatchet or double hatchet flap. (Figures 2 - 4). Gurunluoglu et al. describe its use within the region for primary closure of defects up to $3.5 \mathrm{~cm}$ in maximum diameter while maintaining neurovascular integrity of the region. (Gurunluoglu) This flap design allows for optimal rotation and wound coverage, while minimizing tension and distortion of adjacent subunits. The incisions should be designed in such a manner as to align with the hairline and facial rhytids if possible.

\section{TEMPLE REGION}

The temporal region of the forehead is separated from the lateral border of the paramedian region by the temporal line. The temporal line is an arc of bone, which serves as an attachment point for the temporal fascia. More posteriorly the temporal line divides into a superior and inferior temporal line, the superior corresponding to temporal fascia insertion, and the inferior corresponding to the origin of the temporalis muscle. The temporal line extends from the parietal bone posteriorly to the posterior border of the zygoma at the frontozygomatic suture line. The mid forehead and paramedian regions lie anteromedial to the temporal line and are convex in shape.

The temporal region of the forehead lies posterolateral to the temporal line and is predominantly concave. This concavity affords the reconstructive surgeon different reconstructive options when compared to how one may address the convex regions of the forehead. Additionally, there is a relative increase in subcutaneous tissues due to the volume provided by the temporalis muscle and temporal fat pad.

The temporal region of the forehead is home to several soft tissue structures, including the temporalis muscle. The primary neurovascular structures within the temporal region which are of interest to the reconstructive include the superficial and deep temporal vessels, the frontal branch of the facial nerve, and the sentinel vein. The sentinel vein is a large, zygomaticotemporal perforating vessel between the deep 
temporal fascia and the temporoparietal fascial layers. It lies approximately $1 \mathrm{~cm}$ lateral to the frontozygomatic suture line and sits adjacent to the frontal branch of the facial nerve (Sabini).

The frontal branch of the facial nerve runs through the temporal region and is responsible for nearly all dynamic movement of the forehead region. It innervates both the periorbital musculature, as well as the frontal division of the occipitofrontalis muscle. Its superficial location of the frontal branch of the facial nerve within the temporal region makes it particularly susceptible to injury, even in cases of relatively shallow soft tissue manipulation (Figure 5).

The frontal branch is most at risk for injury as it crosses over the zygoma. The frontal nerve travels in an oblique path. At that level, it lies within the undersurface of the temporoparietal fascia. Several imaginary lines have been described to predict its path across the zygoma. In general, it travels along an imaginary line traveling from the earlobe to $1.5 \mathrm{~cm}$ above the lateral border of the eyebrow (Pitanguay's line). (Pitanguay)

Another method of using topographic localization of the frontal branch is described by Sabini et al. They first drew a line from tragus to lateral canthus. A second line was then drawn from the bottom of the ear lobe in an oblique fashion, bisection the first line. This second line was similar in orientation to Pitanguay's line. It was found to closely associate with the course of the facial nerve, but also eliminate the anatomic variability found in patient's eyebrow position. As the nerve courses medially, it inserts on the deep aspect of the frontalis muscle.

The superficial temporal fascia (STF) layer continues as the superficial musculoaponeurotic system (SMAS) inferiorly, and the galea aponeurosis superiorly. This fascial layer contains key neurovascular structures, including the superficial temporal artery, the auriculotemporal nerve, and the frontal branch of the facial nerve.

Reconstructive options within the temporal region include rotational flaps and advancement flaps. Many options used successfully in other forehead regions can be applied in the temporal region as well. Benefits of this region include its relatively increased laxity and concavity. Due to the concave nature of the temporal region, it is more amenable to skin grafting when other options are insufficient. Skin grafting can also be considered in patients unlikely to tolerate more invasive reconstructive measures. 


\section{SCAR MANAGEMENT SPECIFIC TO THE FOREHEAD}

The first step to a satisfactory outcome with scar revision is a detailed analysis and correct diagnosis of the problem. Scar revision and management involves much more than simply excising a scar and doing a better closure of the wound. Successful scar revision encompasses disguising the fact that a scar is present and tricking the observer's eye into overlooking or ignoring the scar's presence.

Additionally, a wide array of techniques must exist within the surgeon's armamentarium, both surgical and non-surgical, in order to appropriately manage the high variability of scar presentations. The surgeon's goal should be to select the best technique for the individual patient and scar. The "best technique" tends to be subjective given the relative lack of high-level evidence. It is difficult to produce a well-designed, comparison-controlled trials of various interventions because of differences in objective characterization of scars (color, elevation, texture), and lack of appropriate controls.

Scars can be divided into hypertrophic scars/keloids and non-hypertrophic scars (Lee). Hypertrophic scars and keloids are similar in nature and represent an abnormality in wound healing. Histologic findings of hypertrophic scars and keloids include extracellular matrix (ECM) overproduction and deposition of highly disorganized collagen by fibroblasts. Keloids, by definition, are elevated fibrous scars that extend beyond the borders of the wound and do not regress. Hypertrophic scars tend to develop earlier than keloids ( 8 weeks versus 12 weeks), are confined to the wound borders, and regress at 12 to 24 months. Non-hypertrophic scars are divided into atrophic (depressed) scars or scars that are flat.

Factors impacting the development of unnecessary scar development include: the direction of the scar with respect to RSTL's, distortion of facial subunits, irregular texture to the scar surface, and a color mismatch between the scar and surrounding tissues. Aesthetic borders between or at the margins of facial subunits are excellent locations for incision placement or scar relocation. With respect to the forehead region, these borders include the hairline and eyebrow line. Acknowledgement of RSTL's is important because an incision or closure that crosses these at an angle greater than 30 to 40 degrees will produce an aesthetically displeasing result. One of the ideal scar camouflage techniques is achieved by placement of incisions within existing skin folds produced by RSTL's (Clark, Schweinfurth). 


\section{Surgical Techniques}

\section{Hair Transplantation}

Scars within the forehead region may include hair bearing skin. Hair transplantation may be considered in such cases to aid in scar camouflage. As hair transplantation techniques have evolved from large, multi-follicular unit "plugs" to follicular units and single follicle micrografts, successful hair transplantation can be nearly indistinguishable from native hair by the casual observer.

The eyebrow can be restored with hair transplantation. Care is made to align the vector of the transplanted follicle with the natural vector of eyebrow hair. Depending on the donor site, the patient should be counseled on the likely need to trim and maintain the transplanted hairs, as they will continue to grow and function in a manner that is dependent on their site of origin. The process can be time consuming, but good results have been reported. (Goldman)

\section{Z-plasty (Hove)}

The Z-plasty transposition flap can provide excellent cosmesis and is primarily used to lengthen the scar, release scar contracture, disrupt a scar or realign a scar within a RSTL. Z-plasty does have its drawbacks, which include the fact that lengthening a scar in one direction will result in shortening in the perpendicular direction. Also, a total of three scars will be made in place of one. The ultimate effect of a Zplasty is disruption of the scar itself and reorientation of the scar along the limbs of incisions.

W-plasty

The W-plasty is helpful in managing long, straight scars. These scars reflect light homogeneously and are easy for the eye to follow. The $\mathrm{W}$-plasty creates a regularly irregular incision, which allows for light scattering, making the scar less visible to the observer (Figures 6-7). Unlike a Z-plasty, this technique does not increase the length of the scar. 


\section{Geometric Broken Line Closure (GBLC)}

GBLC produces an irregularly, irregular incision and is more complicated and time consuming than a W-plasty. (Figures 8-9) This technique is ideally used for scars which are long and angled 45 degrees or greater from RSTLs. GBLC incorporates a series of advancement flaps in the form of opposing semicircles, squares, rhomboids, triangles, and rectangles in varying order and size. The key feature of this technique is the randomness of its design, which is difficult for the observer to track. Thus, GBLC is useful for lengthy and irregular scars of the forehead. The GBLC design is excised under tension taking the interposing skin and scar.

\section{Non-Surgical and Adjunctive Techniques}

While surgery is the mainstay of treatment for scars in the forehead region, non-surgical and adjunct modalities should be part of the surgeon's armamentarium. These techniques can be used as monotherapies in the appropriate clinical setting. This section will review the various non-surgical and adjunctive techniques to forehead scars.

Steroids

Steroids continue to be a good option for scar management, camouflage and revision in the forehead region. The mechanism of action is believed to be the suppression and reduction of collagen cross-linking and deposition, thus preventing further scar formation. Steroids injected intralesionally have been shown to decrease the elevation and erythema of scars, which can be used immediately after surgical closure and also as a primary intervention. Steroids are used as first-line treatment for both hypertrophic scars and keloids. The most commonly used steroid is triamcinolone acetonide (Kenalog). Triamcinolone injections have been shown to flatten small keloids by $50 \%$ to $100 \%$ and decrease their rate of recurrence (Juckett). The recurrence rates improve more when intralesional steroid injection is coupled with other modalities including silicone sheeting and/or surgical excision. Earlier steroid injections also yield superior outcomes with regard to scar prevention. Side effects of steroid injection include hypopigmentation, pain at site of injection, dermal and adipose atrophy, and rarely, tissue necrosis (Capon). 


\section{Laser Resurfacing}

Lasers have been widely used for scar management (Lee). Lasers can be used as monotherapy or as an adjunct to surgery and other non-surgical techniques. Lasers are divided into ablative and nonablative depending on depth of penetration and based on wavelength and absorption. Both types of lasers play a role in scar management and their use can be used in management of forehead scars. All lasers should be used judiciously in patients with skin types Fitzpatrick IV - VI because of an increased side effect profile, primarily irreversible hypopigmentation.

The two most commonly used ablative lasers are the CO2 laser and the Er:YAG laser. The absorbing chromophore for both lasers is water. Both lasers increase thermal energy which results in vaporization. Ablative lasers are known to yield good results for treating hypertrophic scars and keloids (Figures 10-11). Ablative lasers can be used as monotherapy, but superior results are appreciated when combined with intralesional steroid injection. Er:YAG causes less tissue necrosis than $\mathrm{CO} 2$ and may be better suited for scar margins. Disadvantages to ablative lasers include: pain, prolonged down time, persistent erythema up to 3 months, increased risk for viral and bacterial infections, hyperpigmentation and hypopigmentation. Er:YAG has a lower likelihood of the aforementioned problems compared to CO2 because it can be applied more precisely and has a higher affinity for water. Fractionated $\mathrm{CO} 2$ lasers also have a better safety profile and lesser downtime when compared to traditional CO2 lasers. (Elsaie) It should be noted that some of the benefits of these lasers, namely the elimination of dyschromias, may cause abnormal pigmentation patterns and the treatment of the entire forehead, or even the entire face, may provide the optimal aesthetic results.

Non-ablative lasers (NAL) include the 585-nm pulsed dye laser (PDL) and Nd:YAG laser. The main chromophore is oxyhemoglobin in the microvasculature, which leads to targeted vaporization and subsequent tissue ischemia. Intracellular water is not affected (compared to ablative lasers), thus no tissue necrosis is noted. The net result is a leveling of the scar with decreased erythema. NAL have also been shown to stimulate extracellular matrix (ECM) deposition, which makes these lasers desirable for 
depressed or atrophic scars. NAL are also used to treat hyperpigmented scars. Disadvantages to NAL include downtime, erythema, and an increased risk for viral and bacterial infections. (Alster)

\section{Dermabrasion}

Dermabrasion can be used as a non-surgical treatment for forehead scars. The goal of dermabrasion is to level the skin of a hypertrophic scar or keloid while also promoting re-epithelialization and ECM deposition. Dermabrasion classically has been used for acne scarring and rhinophyma but can be used after surgery to smooth uneven scars. Care must be taken to injure the epithelium to the level of the papillary dermis as the reticular dermis maintains the structures which will promote re-epithelialization.

\section{Intralesional Fillers}

Injectable fillers are very useful for atrophic scars in the forehead region. They function as a means of direct scar camouflage. Fillers can be autologous (fat), allogenic, xenogenic or synthetic. Of note, fillers may elicit a foreign body or inflammatory response after injection which may paradoxically cause hypertrophic changes. Thus, patients must be counseled on the potential for superficial skin changes or granuloma formation. Neurovascular anatomy and patterns in the forehead must be appreciated, as necrosis due to intravascular injection has been described in the forehead region.

\section{Botulinum Toxin}

An increasing body of evidence suggests that botulinum toxin-induced immobilization of forehead wounds results in enhanced wound healing (Sherris). The forehead is a particularly favorable area to treat because of its low associated risk of inducing temporary functional deficits. Botulinum toxin temporarily weakens surrounding muscles, thus lessening the pull on the wound during the acute healing phase of the first 2-4 months. By paralyzing nearby muscles, botulinum toxin helps prevent the development of a wide scar (Figures 12-13). As a general principle, the senior author advises all patients undergoing scar revision in the forehead region receive botulinum toxin injection 2 weeks prior to surgery. 


\section{REFERENCES}

Alster TS, Tanzi EL, Lazarus M. The use of fractional laser photothermolysis for the treatment of atrophic scars. Dermatol Surg 2007;33(3):295-299.

Bryant, Lucas, Ryan Heffelfinger, and Edmund Pribitkin. "Management of Acute Facial Soft Tissue Injuries." Sataloff's Comprehensive Textbook of Otolaryngology: Head \& Neck Surgery (Facial Plastic and Reconstructive Surgery) - Volume 3. Ed. Anthony Sclafani. 1st ed. 3 Vol. Philadelphia: Jaypee, 2016. 1. Print.

Capon A, Iarmarcovai G, Gonnelli D, et al. Scar prevention using laser-assisted skin healing (LASH) in plastic surgery. Aesthetic Plast Surg 2010;34(4):438-446.

Clark JM, Wang TD. Local flaps in scar revision. Facial Plast Surg 2001;17(4):295-308.

Elsaie ML, Choudhary S. Lasers for scars: a review and evidence-based appraisal. J Drugs Dermatol 2010;9(11):1355-1362.

Goldman, Glenn D. "Eyebrow transplantation." Dermatologic surgery 27.4 (2001): 352-354.

Gurunluoglu, R., et al. "Reconstruction of Large Supra-Eyebrow and Forehead Defects using the Hatchet Flap Principle and Sparing Sensory Nerve Branches." Annals of Plastic Surgery 68.1 (2012): 37-42. Web.

Hove CR, Williams EF III, Rodgers BJ. Z-plasty: a concise review. Facial Plast Surg 2001;17(4):289-294.

Igde, M., et al. "Reconstruction of Tissue Defects Developing After Excision of Non-Melanoma Malignant Skin Tumors in Scalp and Forehead Regions." Turkish neurosurgery 25.6 (2015): 888-94. Web.

Juckett G, Hartman-Adams H. Management of keloids and hypertrophic scars. Am Fam Physician 2009;80(3):253-260.

Lee Y. Combination treatment of surgical, post-traumatic and post-herpetic scars with ablative lasers followed by fractional laser and non-ablative laser in Asians. Lasers Surg Med 2009;41(2):131-140.

Levine, Elie, et al. "Quality of life and facial trauma: psychological and body image effects." Annals of plastic surgery 54.5 (2005): 502-510. 
Ozkaya Mutlu, O., et al. "Aesthetic Unit-Based Reconstruction of Periorbital Defects." The Journal of craniofacial surgery 27.2 (2016): 429-32. Web.

Pitanguy I, Ramos AS. "The frontal branch of the facial nerve: the importance of its variations in face lifting."

Plast Reconstr Surg. 1966;38:352-356.

Raposio, E, PierLuigi, S, Nordstrom, RE. "Effects of Galeotomies on Scalp Flaps". Annals of Plastic Surgery. July 1998, 41 (1); 17-21.

Sabini, P., I. Wayne, and V. C. Quatela. "Anatomical Guides to Precisely Localize the Frontal Branch of the Facial Nerve." Archives of facial plastic surgery 5.2 (2003): 150-2. Web.

Schweinfurth JM, Fedok F. Avoiding pitfalls and unfavorable outcomes in scar revision. Facial Plast Surg 2001;17(4):273-278.

Sherris DA, Gassner HG. Botulinum toxin to minimize facial scarring. Facial Plast Surg 2002;18 (1) 3539.

Siegle, Ronald. "Reconstruction of the Forehead." Local Flaps in Facial Reconstruction. Ed. Shan Baker. 3rd ed. Philadelphia, PA: Elsevier Saunders, 2014. 563-588. Print.

\section{FIGURE LEGEND}

Figure 1 - Artistic rendering of forehead subunits (mid-forehead, paramedian, temple).

Figures 2-4 - Artistic rendering of hatchet flap in the paramedian forehead. Note how incision placement allows wound closure in line of the relaxed skin tension lines.

Figure 5 - Artistic rendering of the frontal branch of the facial nerve relative to the fascial layers of the temporal forehead area.

Figure 6 - Preoperative marking of W-plasty in two long, vertical paramedian forehead scars.

Figure 7 - Postoperative picture of a patient after a W-plasty of two paramedian forehead scars.

Figure 8 - Preoperative marking of geometric broken line closure of a paramedian forehead scar. Note the scar traverses the eyebrow. 
Figure 9 - Postoperative picture of a patient after a geometric broken line closure of a paramedian forehead scar.

Figure 10 - Pre-CO2 laser picture of a patient with a raised, erythematous forehead scar.

Figure 11 - Post-CO2 laser picture of a patient with a forehead scar. Note the flattening and depigmentation of the scar.

Figure 12 - Patient with scar in mid-forehead.

Figure 13 - Mid-forehead scar worsens with muscle contraction. This patient is a good candidate for Botox injection. 